\title{
A Numerical Scheme for Stochastic PDEs with Gevrey Regularity
}

\author{
Gabriel J Lord ${ }^{\dagger} \quad$ Jacques Rougemont ${ }^{\ddagger}$ \\ † Department of Mathematics, Heriot-Watt University, Edinburgh EH14 4AS, United Kingdom. \\ $\ddagger$ TAGC, INSERM-ERM 206, Parc scientifique de Luminy, Case 906,13288 Marseille cedex 9, France.
}

November 3, 2003

\begin{abstract}
We consider strong approximations to parabolic stochastic PDEs. We assume the noise lies in a Gevrey space of analytic functions. This type of stochastic forcing includes the case of forcing in a finite number of Fourier modes. We show that with Gevrey noise our numerical scheme has solutions in a discrete equivalent of this space and prove a strong error estimate. Finally we present some numerical results for a stochastic PDE with a GinzburgLandau nonlinearity and compare to the more standard implicit Euler-Maruyama scheme.
\end{abstract}




\section{Introduction}

We consider the numerical approximation of stochastic PDEs of the following type

$$
\dot{u}(t)=\Delta u(t)+F(u(t))+\mathcal{Q} \dot{W}(t),
$$

with periodic boundary conditions on $[0,2 \pi)$ and initial condition $u_{0} \in C_{\mathrm{per}}([0,2 \pi], \mathbb{C})$, the space of continuous periodic functions. The corresponding integral equation is given by

$$
u(t)=e^{t \Delta} u(0)+\int_{0}^{t} e^{(t-s) \Delta} F(u(s)) \mathrm{d} s+\int_{0}^{t} e^{(t-s) \Delta} \mathcal{Q} \mathrm{d} W(s),
$$

where the stochastic integral is taken in Itô's sense (it is a simple exercise to adapt our results to the Stratonovich, or any other case, see the remark after Eq.(3.7)). Here $W$ is a cylindrical Wiener process and $\mathcal{Q}$ a Hilbert-Schmidt operator on Gevery space, namely it has exponentially decaying Fourier coefficients. In this paper, we develop a numerical scheme which preserves the exponential decay of Fourier modes and thereby improve on standard error estimates for Galerkin schemes for stochastic PDES. The precise assumptions on the nonlinearity $F$ and noise $\mathcal{Q} \dot{W}$ are detailed in Sections 2.2-2.3.

We show, in Section 4, that our numerical scheme has solutions in a discrete equivalent of the Gevrey space. There is a long history of the use of Gevrey regularity in deterministic PDEs and in their numerical analysis: the work on Navier-Stokes by [6] was generalized to nonlinear parabolic equations in $[18,5]$, and as a consequence exponential convergence of the Galerkin scheme was obtained in [4]. For stochastic PDEs a number of authors have considered noise $\mathcal{Q} \dot{W}$ which is smooth in space and white noise in time, for example [1, $19,17]$ consider the 2D Navier-Stokes equation and obtain regularity results with smooth forcing and [2] considers Gevrey regularity of attractors for stochastic reaction-diffusion equations. In a similar spirit to these works we choose the noise in the smallest space in which the deterministic part of the PDE is known to have solutions, namely a Gevrey space of analytic functions (see Eq. (2.2)). Naively, we expect that the regularity of the solution is determined by the regularity of the noise, but that it could not be any smoother than for the deterministic case, namely Gevrey. We note that the Gevrey regularity covers the case of a stochastic forcing in a finite number of modes. For the deterministic PDE, Gevrey regularity of numerical solutions based on a Fourier decomposition was considered in this form in [13], whilst different discrete Gevrey spaces were defined in [20], and for finite differences in [14]. The spatial discretisation we consider here is a Galerkin approximation.

Having obtained smoothness of the solutions we exploit this to prove in Section 5 a strong error estimate that improves over standard results in the literature. This is faster than any polynomial, although not the exponential rate found in [4] for the deterministic case. We restrict our analysis to additive noise since in this case existence and uniqueness of solutions is fairly standard (see [3]). Strong convergence of a Galerkin approximation for stochastic PDEs with additive, spatially white noise has been condsidered by few authors. The standard Euler Maruyama scheme was first considered by [7] (see Section 3), and for this scheme a stability restriction is required on the time step (similar to the deterministic case). Strong convergence for the standard implicit Euler-Maruyama scheme is considered in both [11] and [8] which also considers a number of different spatial and time discretizations.

In Section 7 we present numerical results and examine strong convergence for a forced PDE with a Ginzburg-Landau type nonlinearity. We investigate both smooth and non-smooth 
noise and compare this scheme to a standard implicit Euler-Maruyama scheme. Finally we briefly consider the multiplicative noise case numerically.

\section{Definitions and Assumptions}

\subsection{Functional Spaces}

Let $\mathrm{L}_{\text {per }}^{2}([0,2 \pi), \mathbb{C})$ be the Hilbert space of square-integrable complex-valued $2 \pi$-periodic functions, which we identify with $\ell^{2}(\mathbb{Z}, \mathbb{C})$ by Fourier series:

$$
\begin{aligned}
f(x) & =\sum_{n \in \mathbb{Z}} f_{n} e^{i n x} \equiv \sum_{n \in \mathbb{Z}} f_{n} \varphi_{n}(x) \\
\|f\|_{\mathrm{L}^{2}} & =\left(\frac{1}{2 \pi} \int_{0}^{2 \pi}|f(x)|^{2} \mathrm{~d} x\right)^{\frac{1}{2}}=\left(\sum_{n \in \mathbb{Z}}\left|f_{n}\right|^{2}\right)^{\frac{1}{2}} .
\end{aligned}
$$

We also use $\left(\mathrm{L}^{2}-\right)$ Sobolev spaces $\mathrm{H}^{m}(m \in \mathbb{N})$

$$
\|f\|_{\mathrm{H}^{m}}=\left\|(1-\Delta)^{\frac{m}{2}} f\right\|_{\mathrm{L}^{2}}=\left(\sum_{n \in \mathbb{Z}}\left(1+n^{2}\right)^{m}\left|f_{n}\right|^{2}\right)^{\frac{1}{2}} .
$$

The Hilbert space that we will use most often in this paper is $\mathcal{H}=\mathrm{H}^{1}$. We next define Gevrey spaces $\mathrm{G}_{\alpha}(\alpha>0)$ of analytic functions

$$
\|f\|_{\mathrm{G}_{\alpha}}=\left\|e^{\alpha \sqrt{-\Delta}} f\right\|_{\mathcal{H}}=\left(\sum_{n \in \mathbb{Z}} e^{2 \alpha|n|}\left(1+n^{2}\right)\left|f_{n}\right|^{2}\right)^{\frac{1}{2}} .
$$

The actual Hilbert spaces $\mathrm{H}^{m}$ and $\mathrm{G}_{\alpha}$ are defined as the domains (in $\mathrm{L}_{\text {per }}^{2}([0,2 \pi), \mathbb{C})$ ) of the operators $(1-\Delta)^{\frac{m}{2}}$ and $(1-\Delta)^{\frac{1}{2}} \exp (\alpha \sqrt{-\Delta})$, with scalar products corresponding to the norms (2.1)-(2.2).

Throughout the paper, we use the following notation: if $u \in \mathrm{L}_{\text {per }}^{2}([0,2 \pi), \mathbb{C})$ and $F(u) \in$ $\mathrm{L}_{\text {per }}^{2}([0,2 \pi), \mathbb{C})$, we write $F_{n}(u)$ for the $n$th coefficient in the Fourier series of $F(u)$. The integer part of $x$ is written $[x]$ :

$$
[x]=\max \{n \in \mathbb{Z}: n \leq x\} .
$$

We denote by $\mathrm{P}^{\mathrm{N}}$ the projection onto $\operatorname{span}\left\{\varphi_{-\mathrm{N}}, \ldots, \varphi_{\mathrm{N}}\right\}$, the first $2 \mathrm{~N}+1$ Fourier modes.

\subsection{Nonlinear Term}

We assume two types of Lipschitz conditions on the nonlinear term $F$. The first of these is in $C_{\text {per }}([0,2 \pi], \mathbb{C})$ : there is a polynomial function $B$ on $[0, \infty)^{2}$ such that

$$
|F(x)-F(y)| \leq B(|x|,|y|)|x-y| .
$$


The second of these is in the discrete Gevrey space (see Eq.(2.2)): there is an $\alpha>0$ and a continuous function $B$ on $[0, \infty)^{2}$ such that

$$
\|F(u)-F(v)\|_{\mathrm{G}_{\alpha}} \leq B\left(\|u\|_{\mathrm{G}_{\alpha}},\|v\|_{\mathrm{G}_{\alpha}}\right)\|u-v\|_{\mathrm{G}_{\alpha}} .
$$

In addition to these Lipschitz conditions we assume that the nonlinearity $F$ is polynomially bounded, that is there are positive constants $C$ and $r$ such that

$$
|x|\left|F^{\prime}(x)\right|+|F(x)| \leq C\left(1+|x|^{r}\right) .
$$

Finally we assume that $F$ is dissipative in $C_{\text {per }}([0,2 \pi], \mathbb{C})$, that is, there is an $\eta \in \mathbb{R}$ such that

$$
\operatorname{Re}(\overline{(F(x)-F(y))}(x-y)) \leq \eta|x-y|^{2} .
$$

These assumptions hold for the Ginzburg-Landau nonlinearity $F_{\mathrm{GL}}(u)=-|u|^{2} u$ (see [20] for Eq.(2.3)), and this is the example we consider in Section 7. Dissipativity assumptions usually require $\eta<0$ in Eq.(2.5). This can be achieved by changing the definition of the linear operator: let $L=\Delta+2 \eta$ and $G(x)=F(x)-2 \eta x$. Then Eq. (1.1) is the same as

$$
\dot{u}(t)=L u(t)+G(u(t))+\mathcal{Q} \dot{W}(t),
$$

where the nonlinearity $G$ satisfies a bound like (2.5) with a negative constant and the linear operator $L$ generates a compact quasi-bounded semi-group, hence the existence of solutions follows from standard results (see 3.1). We consider here the regularity and strong numerical approximation of solutions.

\subsection{Noise Term}

Let $W$ be the cylindrical Wiener process on $\mathcal{H}$ and $\mathcal{Q}$ a Hilbert-Schmidt operator over $\mathrm{G}_{\alpha}$. We restrict ourselves to the following type of noise terms:

$$
\mathcal{Q} \dot{W}(t)=\sum_{n \in \mathbb{Z}} b_{n} \varphi_{n} \dot{\beta}_{n}(t), \text { with } \sum_{n \in \mathbb{Z}} e^{2 \alpha|n|}\left(1+n^{2}\right)\left|b_{n}\right|^{2}<\infty,
$$

where $b_{n} \in \mathbb{C},\left\{\beta_{n} \in \mathbb{R}: n \in \mathbb{Z}\right\}$ are mutually independent ordinary Brownian motions and $\varphi_{n}(x)=\exp ($ inx $)$. We also assume $b_{0}=0$. Without loss of generality, we may assume that $\alpha$ in this section and $\alpha$ in Section 2.2 are the same number. We often use the notation $b(x)$ for the function $\sum_{n} b_{n} \varphi_{n}(x)$. In Section 7, Figure 1, we plot a sample path for noise that satisfies (2.6).

We make the assumption that $\Delta$ (the linear operator in Eq.(1.1)) and $\mathcal{Q}$ can be diagonalised simultaneously. This greatly simplifies the notation, but is not crucial.

\section{Numerical Scheme}

We next describe our numerical scheme to integrate Eq.(1.1). Schemes similar to this were considered for the deterministic case in $[20,13]$. We first take Fourier series and obtain the infinite system of coupled equations

$$
u_{n}(t)=e^{-t n^{2}} u_{n}(0)+\int_{0}^{t} e^{-(t-s) n^{2}} F_{n}(u(s)) \mathrm{d} s+\int_{0}^{t} e^{-(t-s) n^{2}} b_{n} \mathrm{~d} \beta_{n}(s), \quad n \in \mathbb{Z},
$$


where we recall that $F_{n}(u)$ is the nth Fourier coefficient of $F(u)$. Let $\mathrm{h}>0$ denote the time step and $\mathrm{N}$ the size of the Galerkin truncation. We consider the discretisation of Eq.(1.1) given by

$$
u_{n}^{\mathrm{N}}((k+1) \mathrm{h})=e^{-\mathrm{h} n^{2}}\left(u_{n}^{\mathrm{N}}(k \mathrm{~h})+\mathrm{h} F_{n}\left(u^{\mathrm{N}}(k \mathrm{~h})\right)+b_{n} \sqrt{\mathrm{h}} X_{k+1, n}\right),
$$

where $n \in\{-\mathrm{N}, \ldots, \mathrm{N}\}$. The noise terms $\left\{X_{k, n}: n \in\{-\mathrm{N}, \ldots, \mathrm{N}\}, k=1, \ldots,[T / \mathrm{h}]\right\}$ are defined by

$$
X_{k, n}=\mathrm{h}^{-1 / 2}\left(\beta_{n}(k \mathrm{~h})-\beta_{n}((k-1) \mathrm{h})\right)
$$

and are (by definition of the Brownian motions) i.i.d. $\mathcal{N}(0,1)$ random variables. In Section 6 we discuss how higher order methods may be obtained and in Section 7 implementation of the scheme is considered. In particular, we compare Eq.(3.2) with the classical EulerMaruyama approximation [12, Sec. 9.1]:

$$
u_{n}^{\mathrm{N}}((k+1) \mathrm{h})=\left(1-\mathrm{h} n^{2}\right) u_{n}^{\mathrm{N}}(k \mathrm{~h})+\mathrm{h} F_{n}\left(u^{\mathrm{N}}(k \mathrm{~h})\right)+b_{n} \sqrt{\mathrm{h}} X_{k+1, n}
$$

and to the implicit Euler-Maruyama scheme [12, Sec. 12.2]

$$
u_{n}^{\mathrm{N}}((k+1) \mathrm{h})=\left(1+\mathrm{h} n^{2}\right)^{-1}\left(u_{n}^{\mathrm{N}}(k \mathrm{~h})+\mathrm{h} F_{n}\left(u^{\mathrm{N}}(k \mathrm{~h})\right)+b_{n} \sqrt{\mathrm{h}} X_{k+1, n}\right) .
$$

Notice that the corrections to Eq.(3.4) introduced in Eq.(3.2) are non-uniform in $n$ :

$$
e^{-\mathrm{h} n^{2}}=\left(1-\mathrm{h} n^{2}\right)+\mathcal{O}\left(n^{4} \mathrm{~h}^{2}\right) .
$$

The relationship between Eq.(3.2) and Eq.(3.1) is quite obvious when we iterate Eq.(3.2):

$$
u_{n}^{\mathrm{N}}(t)=e^{-t n^{2}} u_{n}^{\mathrm{N}}(0)+\sum_{k=1}^{[t / \mathrm{h}]} e^{-(t-(k-1) \mathrm{h}) n^{2}}\left(\mathrm{~h} F_{n}\left(u^{\mathrm{N}}((k-1) \mathrm{h})\right)+b_{n} \sqrt{\mathrm{h}} X_{k, n}\right) .
$$

The discrete scheme is only defined for $t / h \in \mathbb{N}$, but the above formula makes sense for any $t>0$ : it is the solution at time $t \in(k \mathrm{~h},(k+1) \mathrm{h}]$ of the linear differential equation

$$
\begin{aligned}
\dot{u}^{\mathrm{N}}(t) & =\Delta u^{\mathrm{N}}(t), \\
u^{\mathrm{N}}(k \mathrm{~h}+0) & =u^{\mathrm{N}}(k \mathrm{~h})+\mathrm{hP}^{\mathrm{N}} F\left(u^{\mathrm{N}}(k \mathrm{~h})\right)+\sqrt{\mathrm{h}} e^{\mathrm{h} \Delta} \mathcal{Q} X^{\mathrm{N}}(k) .
\end{aligned}
$$

The second sum in Eq.(3.6) is indeed an approximation to the Itô stochastic integral and it is easy at this point to modify the scheme to approximate any another type of stochastic integral. See also Section 6 for higher order schemes.

Proposition 3.1 Eq.(1.1) has a unique $\mathcal{H}$-strong solution $u(t)$ on $[0, T]$ with

$$
\sup _{0 \leq t \leq T} \mathbf{E}\left(\|u(t)\|_{\mathcal{H}}^{2}\right)<\infty
$$

Proof. The Laplacian operator $\Delta$ generates an analytic compact semi-group on $C_{\text {per }}([0,2 \pi], \mathbb{C})$. This and the dissipativity of $F$ (in $C_{\text {per }}([0,2 \pi], \mathbb{C}$ ), see Eq.(2.5)) imply existence and uniqueness in $C_{\text {per }}([0,2 \pi], \mathbb{C})$ by Theorem 7.13 in [3].

To extend this result to $\mathcal{H}$, we only need to remark that the heat kernel $G_{t}(x)$ satisfies $\int_{0}^{T}\left\|\nabla G_{t}\right\| \mathrm{d} t<\infty$ in dimension 1 . Hence applying $\nabla$ to Eq.(1.2) and using $C_{\text {per }}([0,2 \pi], \mathbb{C})$ bounds and Eq.(2.4), we get a bound in $\mathcal{H}$.

The first main result of this paper is a regularity result: solutions of Eq.(3.2) are in a Gevrey space of analytic functions. 
Theorem 3.2 For all $T>0$, there exists a positive random variable $\gamma$ and constants $c, C_{m}$, and $R$ such that

$$
\mathbf{E}\left(\sup _{t \leq T} \exp \left(c\|u(t)\|_{\mathrm{G}_{\gamma}}^{2}\right)\right) \leq R, \quad \mathbf{E}\left(\gamma^{-m}\right) \leq C_{m}, \forall m>0 .
$$

Proof. This Theorem will be restated as Corollary 4.5 in Section 4.

Remark that similar results for the stochastic PDE (1.1) can be found in the literature $[19,1]$ and $[16$, Ch. 5], although this has not been considered for numerical approximations to our knowledge. For the deterministic equation a number of regularity results exist for the numerical approximations. The scheme considered in [13] is similar to the case considered here and Gevrey regularity is proved. The scheme considered in [20] is different and employs a different definition of discrete Gevrey spaces. Our techniques could be applied to the scheme and functional setup of [20], but we feel our approach is notationally simpler and more intuitive.

This regularity result on the numerical scheme allows us to prove (see Section 5) the following strong error estimate.

Proposition 3.3 Eq.(3.6) converges strongly in $\mathcal{H}$ to a solution of Eq.(1.1) as $\mathrm{N} \rightarrow \infty$ and $\mathrm{h} \rightarrow 0$. If $u_{0} \in \mathrm{G}_{\alpha}$, then we have the error estimate

$$
\mathbf{E}\left(\sup _{t \in(0, T]}\left\|u(t)-u^{\mathrm{N}}(t)\right\|_{\mathcal{H}}\right) \leq K(T, m)\left(\mathrm{N}^{-m}+\mathrm{h}\right),
$$

for all $m>0$.

Proof. This is considered in Section 5.

This is an improvement over the results for the standard Euler-Maruyama Eq.(3.4), for which the error bound is

$$
\mathbf{E}\left(\left\|u(t)-u^{\mathrm{N}}(t)\right\|_{\mathcal{H}}\right) \leq K(t)\left(\mathrm{N}^{-1}+\mathrm{N}^{5} \mathrm{~h}\right)
$$

see [7]. It must be said, however, that for our result we have made restrictive assumptions on the noise (Gevrey class of regularity), which is not the case in [7]. The rate of convergence in $\mathrm{N}$ cannot be expected to be faster than the rate of decay of $b_{n}$. Strong convergence for the implicit Euler-Maruyama scheme has been considered for Galerkin approximations of SPDEs by [8] (who also examines other discretisations in space and time) and in [11].

\section{Smoothing Action of the Semi-Flow}

In this section, we explore the contraction properties of the semi-flow generated by Eq.(3.6) in various spaces. Let $\mathcal{F}=\left\{\mathcal{F}_{m}: m \in \mathbb{Z}\right\}$ denote the nonlinear term of Eq.(3.6):

$$
\mathcal{F}_{m}(v)(t)=\sum_{k=1}^{[t / \mathrm{h}]} e^{-(t-(k-1) \mathrm{h}) m^{2}} \mathrm{~h} F_{m}(v((k-1) \mathrm{h}))
$$


acting on the metric space

$$
\mathcal{B}_{R, \gamma}(\tau)=\left\{v:[0, \tau] \times[0,2 \pi) \rightarrow \mathbb{R} ;\|v\|_{\gamma} \equiv \sup _{t \in[0, \tau]}\|v(t)\|_{\mathrm{G}_{\gamma t}} \leq R\right\} .
$$

We prove a contraction result for $\mathcal{F}$ in the following lemma, from which all the results of Section 3 will follow.

Lemma 4.1 For all $R>0$ and $0<\gamma<\alpha$, where $\alpha$ is such that Eq.(2.3) holds, there is a $\tau>0$ such that the map $\mathcal{F}$ is a contraction on $\mathcal{B}_{R, \gamma}(\tau)$.

Proof. Let $v_{1}$ and $v_{2}$ be such that $\left\|v_{1,2} \mid\right\|_{\gamma} \leq R$. First note that

$$
\sum_{k=1}^{[t / \mathrm{h}]} \mathrm{h}\left(\sum_{|n| \leq \mathrm{N}} e^{-(t-(k-1) \mathrm{h}) n^{2}}\right)^{1 / 2} \leq \sqrt{\pi} \sum_{k=1}^{[t / \mathrm{h}]} \mathrm{h}(t-(k-1) \mathrm{h})^{-1 / 2} \leq \sqrt{\pi t} .
$$

Using these estimates, we compute the Lipschitz constant of $\mathcal{F}$ in $\mathcal{B}_{R, \gamma}(\tau)$ :

$$
\begin{aligned}
& \sum_{|n| \leq \mathrm{N}} e^{2 \gamma t|n|}\left(1+n^{2}\right)\left|\mathcal{F}_{n}\left(v_{1}\right)(t)-\mathcal{F}_{n}\left(v_{2}\right)(t)\right|^{2} \\
& =\sum_{|n| \leq \mathrm{N}} \mid \sum_{k=1}^{[t / \mathrm{h}]} \mathrm{h} e^{-(t-(k-1) \mathrm{h}) n^{2}} e^{\gamma(t-(k-1) \mathrm{h})|n|} \\
& \left.e^{\gamma(k-1) \mathrm{h}|n|} \sqrt{1+n^{2}}\left(F_{n}\left(v_{1}((k-1) \mathrm{h})\right)-F_{n}\left(v_{2}((k-1) \mathrm{h})\right)\right)\right|^{2} \\
& \leq\left(\sum_{k=1}^{[t / \mathrm{h}]} \mathrm{h}\left(\sum_{|n| \leq \mathrm{N}} e^{2(t-(k-1) \mathrm{h})|n|(\gamma-|n|)}\right)^{1 / 2} B(R)\left\|v_{1}((k-1) \mathrm{h})-v_{2}((k-1) \mathrm{h})\right\|_{\mathrm{G}_{\gamma(k-1) \mathrm{h}}}\right)^{2} \\
& \leq B^{2}(R)\left\|\mid v_{1}-v_{2}\right\|_{\gamma}^{2}\left(\sum _ { k = 1 } ^ { [ t / \mathrm { h } ] } \mathrm { h } \left(\sum_{|n| \leq \mathrm{N}} e^{\left.2(t-(k-1) \mathrm{h})|n|(\gamma-|n|))^{1 / 2}\right)^{2}}\right.\right. \\
& \leq B^{2}(R) \mid\left\|v_{1}-v_{2}\right\|_{\gamma}^{2}\left(\sum_{k=1}^{[t / \mathrm{h}]} \mathrm{h}\left(\sum_{|n| \leq \mathrm{N}} e^{(t-(k-1) \mathrm{h})\left(\gamma^{2}-n^{2}\right)}\right)^{1 / 2}\right)^{2} \\
& \leq \pi B^{2}(R) e^{\gamma^{2} \tau} \tau\left|\left\|v_{1}-v_{2} \mid\right\|_{\gamma}^{2},\right.
\end{aligned}
$$

where $B(R)=\sup _{x, y<R} B(x, y)$ with $B$ from Eq.(2.3).

By taking the sup over $t<\tau$, we obtain the estimate

$$
\left\|\mathcal{F}\left(v_{1}\right)-\mathcal{F}\left(v_{2}\right)\right\|_{\gamma} \leq \pi B(R) e^{\gamma^{2} \tau} \sqrt{\tau}\left\|v_{1}-v_{2}\right\|_{\gamma} \leq(1-\delta)\left\|v_{1}-v_{2}\right\|_{\gamma},
$$

where $\delta>0$ if $\sqrt{\tau} e^{\gamma^{2} \tau}<1 /(2 B(R))$.

Remark here that the inverse of the maximal Gevrey index, $(\gamma \tau)^{-1}$, is bounded by a polynomial in $R$. This will be used in the proof of Corollary 4.5. 
We now consider the noise term in Eq.(3.6) which we denote $h(t)$ :

$$
h_{n}(t)=\sum_{k=1}^{[t / \mathrm{h}]} e^{-(t-(k-1) \mathrm{h}) n^{2}} b_{n} \sqrt{\mathrm{h}} X_{k, n},
$$

and $g(t)$ the linear part of the evolution, for the initial condition $u_{0}^{\mathrm{N}} \in C_{\mathrm{per}}([0,2 \pi))$ :

$$
g_{n}(t)=e^{-t n^{2}} u_{n}^{\mathrm{N}}(0) .
$$

To use Lemma 4.1 to deduce properties of solutions of Eq.(3.2), we need estimates on the processes $g(t)$ and $h(t)$.

Lemma 4.2 Given $C>0$ there is a $c>0$ such that

$$
\mathbf{E}\left(\sup _{t \leq 1} \exp \left(c\|h(t)\|_{\mathrm{G}_{\alpha}}^{2}\right)\right) \leq C,
$$

where $\alpha$ is such that Eq.(2.6) holds.

Proof. Let $Y_{k, m}=\exp \left(-(t-(k-1) \mathrm{h}) m^{2}\right) \sqrt{\mathrm{h}} X_{k, m}$. Remark that the $Y_{k, m}$ (for simplicity of notation, we set $\left.Y_{k, 0}=0\right)$ are mutually independent $\mathcal{N}\left(0, \mathrm{~h} \exp \left(-2(t-(k-1) \mathrm{h}) m^{2}\right)\right)$ random variables. Therefore, for any $t \geq t_{1}>t_{0}+\mathrm{h}>t_{0} \geq 0$, the sum $\sum_{k=\left[t_{0} / \mathrm{h}\right]+1}^{\left[t_{1} / \mathrm{h}\right]} Y_{k, m}$ is a Gaussian random variable with variance

$$
\begin{aligned}
\sum_{k=\left[t_{0} / \mathrm{h}\right]+1}^{\left[t_{1} / \mathrm{h}\right]} \operatorname{Var}\left(Y_{k, m}\right) & =\mathrm{h} e^{-2 t m^{2}} \sum_{k=\left[t_{0} / \mathrm{h}\right]}^{\left[t_{1} / \mathrm{h}\right]-1} e^{2 k \mathrm{~h} m^{2}} \\
& =\mathrm{h} e^{-2 t m^{2}}\left(\frac{e^{2\left[t_{1} / \mathrm{h}\right] \mathrm{h} m^{2}}-e^{2\left[t_{0} / \mathrm{h}\right] \mathrm{h} m^{2}}}{e^{2 \mathrm{~h} m^{2}}-1}\right) \\
& \leq\left(\left[t_{1} / \mathrm{h}\right]-\left[t_{0} / \mathrm{h}\right]\right) \mathrm{h} .
\end{aligned}
$$

Therefore, given $C>0$, for $c_{0}$ sufficiently small we get

$$
\mathbf{E}\left(\exp \left(c_{0}\left|\sum_{k=\left[t_{0} / \mathrm{h}\right]}^{\left[t_{1} / \mathrm{h}\right]} Y_{k, m}\right|^{2} /\left(t_{1}-t_{0}\right)\right)\right) \leq C .
$$

We now choose the constant $c$ such that

$$
c\|b\|_{\mathrm{G}_{\alpha}}^{2} \equiv c \sum_{n \in \mathbb{Z}} e^{2 \alpha|n|}\left(1+n^{2}\right)\left|b_{n}\right|^{2} \leq c_{0} .
$$

Then, using $\mathbf{E}\left(X^{q}\right) \leq(\mathbf{E}(X))^{q}$ for $q \leq 1$ (Jensen's inequality) and mutual independence of the random variables, we get

$$
\begin{aligned}
\mathbf{E} & \left(\exp \left(c\left\|h\left(t_{1}\right)-e^{\left(t_{1}-t_{0}\right) \Delta} h\left(t_{0}\right)\right\|_{\mathrm{G}_{\alpha}}^{2} /\left(t_{1}-t_{0}\right)\right)\right) \\
& =\mathbf{E}\left(\exp \left(\sum_{m \in \mathbb{Z}} c e^{2 \alpha|m|}\left(1+m^{2}\right)\left|b_{m}\right|^{2}\left|\sum_{k=\left[t_{0} / \mathrm{h}\right]+1}^{\left[t_{1} / \mathrm{h}\right]} Y_{k, m}\right|^{2} /\left(t_{1}-t_{0}\right)\right)\right)
\end{aligned}
$$




$$
\begin{aligned}
& =\prod_{m \in \mathbb{Z}} \mathbf{E}\left(\exp \left(c e^{2 \alpha|m|}\left(1+m^{2}\right)\left|b_{m}\right|^{2}\left|\sum_{k=\left[t_{0} / \mathrm{h}\right]+1}^{\left[t_{1} / \mathrm{h}\right]} Y_{k, m}\right|^{2} /\left(t_{1}-t_{0}\right)\right)\right) \\
& \leq \prod_{m \in \mathbb{Z}}\left(\mathbf{E}\left(\exp \left(c\|b\|_{\mathrm{G}_{\alpha}}^{2}\left|\sum_{k=\left[t_{0} / \mathrm{h}\right]+1}^{\left[t_{1} / \mathrm{h}\right]} Y_{k, m}\right|^{2} /\left(t_{1}-t_{0}\right)\right)\right)\right)^{\exp (2 \alpha|m|)\left(1+m^{2}\right)\left|b_{m}\right|^{2}\|b\|_{\mathrm{G}_{\alpha}}^{-2}} \\
& \leq C
\end{aligned}
$$

We can write the noise term $h(t)$ from (3.6) as

$$
\begin{aligned}
h(t)= & h(t)-e^{\left(t-t_{n}\right) \Delta} h\left(t_{n}\right)+e^{\left(t-t_{n}\right) \Delta}\left(h\left(t_{n}\right)-e^{\left(t_{n}-t_{n-1}\right) \Delta} h\left(t_{n-1}\right)\right)+\cdots \\
& +e^{\left(t-t_{1}\right) \Delta}\left(h\left(t_{1}\right)-e^{t_{1} \Delta} h(0)\right)+e^{t \Delta} h(0),
\end{aligned}
$$

(note that $h(0)=0$ ) for an arbitrary choice of $0<t_{1}<\cdots<t_{n}<t$. Each term in this sum satisfies an inequality like Eq.(4.1), hence the claim follows from an application of Kolmogorov's maximal inequality [10].

We now consider $g(t)$ from the linear part of the evolution.

Lemma 4.3 For all $\gamma>0$,

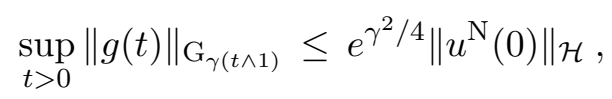

(where $t \wedge 1=\min (t, 1)$ ).

Proof. This is an elementary calculation:

$$
\begin{aligned}
\|g(t)\|_{\mathrm{G}_{\gamma(t \wedge 1)}}^{2} & =\sum_{n \in \mathbb{Z}} e^{2 \gamma|n|(t \wedge 1)-2 n^{2} t}\left(1+n^{2}\right)\left|u_{n}^{\mathrm{N}}(0)\right|^{2} \\
& \leq e^{\gamma^{2}(t \wedge 1)^{2} /(2 t)} \sum_{n \in \mathbb{Z}}\left(1+n^{2}\right)\left|u_{n}^{\mathrm{N}}(0)\right|^{2} \\
& \leq e^{\gamma^{2} / 2}\left\|u^{\mathrm{N}}(0)\right\|_{\mathcal{H}}^{2} . \square
\end{aligned}
$$

To obtain the regularity result we introduce a new map $\widetilde{\mathcal{F}}^{g, h}$ defined as follows:

$$
\widetilde{\mathcal{F}}^{g, h}(f)=\mathcal{F}(f+g+h) .
$$

Obviously, if $\widetilde{f}$ is a fixed point of $\widetilde{\mathcal{F}}^{g, h}$, then $\widetilde{f}+g+h$ is a solution of Eq.(3.2).

Lemma 4.4 Let $\alpha$ be such that Eq.(2.3) holds and let $h \in \mathcal{B}_{R, \gamma}(1)$ for some $0<\gamma<\alpha$. Then $\widetilde{\mathcal{F}}^{g, h}$ has a unique fixed point in $\mathcal{B}_{R, \gamma}(1)$.

Proof. By Eq.(2.2) and Lemmas 4.2-4.3, there is a $C$ such that $\sup _{t \leq 1}\|g(t)+h(t)\|_{\mathrm{G}_{\alpha}}<C$, thus $g+h$ is in $\mathcal{B}_{C, \alpha}(1)$ hence $\widetilde{\mathcal{F}}^{g, h}$ is well-defined on $\mathcal{B}_{C, \alpha}(\tau)$ for $\tau<1$.

Lemma 4.1 shows that $\widetilde{\mathcal{F}}^{g, h}$ is a contraction for $\tau$ small enough, hence by the Banach fixed point Theorem, it has a fixed point. The result can be iterated to get a fixed point in $\mathcal{B}_{C, \alpha}(1)$.

We next apply this result to the random process defined by Eq.(3.2): 
Corollary 4.5 Let $u(t)$ be defined by Eq.(3.2) with $u_{0} \in \mathrm{G}_{\alpha}$. For all $T>0$, there exists $a$ random variable $\gamma$ and constants $c, C_{m}$ and $R$ such that

$$
\mathbf{E}\left(\sup _{t \leq T} \exp \left(c\|u(t)\|_{\mathrm{G}_{\gamma}}^{2}\right)\right) \leq R, \quad \mathbf{E}\left(\gamma^{-m}\right) \leq C_{m}, \forall m>0 .
$$

Proof. By Lemma 4.4, since $\tau>0$ almost surely, $u$ satisfies Eq.(4.2) with $R=R_{0} \mathbf{E}\left(\exp \left(c\|h\|_{\mathrm{G}_{\gamma}}^{2}\right)\right)$ provided $h \in \mathrm{G}_{\gamma}$, which is true almost surely by Lemma 4.2, and $\gamma$ is such that Lemma 4.4 holds in $\mathcal{B}_{\|h\|_{\alpha}, \gamma}(t)$. The bound on the distribution of $\gamma$ follows from the fact that $\gamma^{-1}$ can bounded by a polynomial in $\|h\|_{\mathrm{G}_{\gamma}}$ (see remark at the end of the proof of Lemma 4.4) since

$$
\mathbf{E}\left(\gamma^{-m}\right) \leq \mathbf{E}\left(\|h\|_{\alpha}^{C(m)}\right) \leq C(m) \mathbf{E}\left(\sup _{t<T} e^{c\|h(t)\|_{\mathrm{G}_{\alpha}}^{2}}\right)
$$

\section{Strong Error Estimate}

We want to estimate the strong error of Proposition 3.3

$$
\mathbf{E}\left(\sup _{t \in(0, T]}\left\|u(t)-u^{\mathrm{N}}(t)\right\|_{\mathcal{H}}^{2}\right)
$$

where $u(t)$ is the solution of Eq.(1.2) and $u^{\mathrm{N}}(t)$ the solution of Eq.(3.2) with the same initial condition $u_{0}$ (see [12], p.324-325).

We split the problem into Fourier modes with $|n| \leq \mathrm{N}$ and $|n|>\mathrm{N}$ and consider the non-linear and noise terms separately. First we look at the non-linear term.

$$
\begin{gathered}
\mathbf{E}\left(\sup _{t \in(0, \tau]} \sum_{|n| \leq \mathrm{N}}\left|\sum_{k=1}^{[t / h]} \int_{(k-1) \mathrm{h}}^{k \mathrm{~h}} e^{-(t-(k-1) \mathrm{h}) n^{2}}\left(1+n^{2}\right)\left(e^{(s-(k-1) \mathrm{h}) n^{2}} F_{n}(u(s))-F_{n}\left(u^{\mathrm{N}}\left(t_{k}\right)\right)\right) \mathrm{d} s\right|^{2}\right) \\
\leq \sum_{|n| \leq \mathrm{N}} \mathbf{E}\left(\operatorname { s u p } _ { t \in ( 0 , \tau ] } \sum _ { k = 1 } ^ { [ t / h ] } \int _ { ( k - 1 ) \mathrm { h } } ^ { k \mathrm { h } } e ^ { - ( t - ( k - 1 ) \mathrm { h } ) n ^ { 2 } } ( 1 + n ^ { 2 } ) \left(\left|F_{n}\left(u^{\mathrm{N}}(s)\right)-F_{n}\left(u^{\mathrm{N}}\left(t_{k}\right)\right)\right|\right.\right. \\
\left.\left.+\left|F_{n}(u(s))-F_{n}\left(u^{\mathrm{N}}(s)\right)\right|+\left|\left(e^{(s-(k-1) \mathrm{h}) n^{2}}-1\right) F_{n}(u(s))\right|\right) \mathrm{d} s\right)^{2}
\end{gathered}
$$

We use the bounds on the nonlinearity Eq.(2.3)-(2.4)

$$
\begin{aligned}
& \leq C\left(\tau^{2} \mathrm{~h}^{2}+\int_{0}^{\tau} \mathbf{E}\left(\sup _{s \in(0, t]}\left\|F(u(s))-F\left(u^{\mathrm{N}}(s)\right)\right\|_{\mathcal{H}}^{2}\right) \mathrm{d} t+\mathbf{E}\left(\tau^{2} \mathrm{~h}^{2} \sup _{s \in(0, \tau]}\|u(s)\|_{\mathrm{G}_{\gamma}}^{2 r}\right)\right) \\
& \leq C\left(\tau^{2} \mathrm{~h}^{2}+\int_{0}^{\tau} \mathbf{E}\left(\sup _{s \in(0, t]}\left\|u(s)-u^{\mathrm{N}}(s)\right\|_{\mathcal{H}}^{2}\right) \mathrm{d} t\right) .
\end{aligned}
$$


Similarly, for $|n|>$ N, using Eq.(2.4), for $m>0$

$$
\begin{aligned}
& \mathbf{E}\left(\sup _{t \in(0, \tau]} \sum_{|n|>\mathrm{N}}\left|\int_{0}^{t} e^{-(t-s) n^{2}}\left(1+n^{2}\right) F_{n}(u(s)) \mathrm{d} s\right|^{2}\right) \\
& \leq C_{m} \mathrm{~N}^{-m} \mathbf{E}\left(N^{2 m} e^{-2 \gamma N}\right)^{1 / 2} \mathbf{E}\left(\sup _{s \in(0, \tau]}\|F(u(s))\|_{\mathrm{G}_{\gamma}}^{4}\right)^{1 / 2} \\
& \leq C_{m} \mathrm{~N}^{-m} \mathbf{E}\left(\sup _{s \in(0, \tau]}\|u(s)\|_{\mathrm{G}_{\gamma}}^{4 r}\right)^{1 / 2}
\end{aligned}
$$

where $\gamma$ is as in Theorem 3.2.

Next the noise contribution is analysed: using Doob's martingale inequality [15] as in [9], we get

$$
\begin{aligned}
& \mathbf{E}\left(\sup _{t \in(0, \tau]} \sum_{|n| \leq \mathrm{N}}\left(1+n^{2}\right)\left|\sum_{k=1}^{[t / h]}\left(\int_{(k-1) \mathrm{h}}^{k \mathrm{~h}} e^{-(t-s) n^{2}} b_{n} \mathrm{~d} \beta_{n}(s)-e^{-(t-(k-1) \mathrm{h}) n^{2}} b_{n} \sqrt{\mathrm{h}} X_{k, n}\right)\right|^{2}\right) \\
& \leq 4 \sum_{|n| \leq \mathrm{N}}\left(1+n^{2}\right)\left|b_{n}\right|^{2} \mathbf{E}\left(\int_{0}^{\tau}\left(e^{-(\tau-s) n^{2}}-e^{-(\tau-[s / h]) \mathrm{h}) n^{2}}\right) \mathrm{d} \beta_{n}(s)\right)^{2} \\
& =4 \sum_{|n| \leq \mathrm{N}}\left(1+n^{2}\right) e^{2 \alpha|n|}\left|b_{n}\right|^{2} \int_{0}^{\tau}\left(e^{-(\tau-s) n^{2}}-e^{-(\tau-[s / h]) \mathrm{h}) n^{2}}\right)^{2} e^{-2 \alpha|n|} \mathrm{d} s \\
& \leq C \tau \mathrm{h}^{2}\|b\|_{\mathrm{G}_{\alpha}}^{2},
\end{aligned}
$$

where $\|b\|_{\mathrm{G}_{\alpha}}^{2}=\sum_{n}\left(1+n^{2}\right) e^{2 \alpha|n|}\left|b_{n}\right|^{2}$, and for $|n|>\mathrm{N}$,

$$
\mathbf{E}\left(\sup _{t \in(0, \tau]} \sum_{|n|>\mathrm{N}}\left(1+n^{2}\right)\left|\int_{0}^{t} e^{-(t-s) n^{2}} b_{n} \mathrm{~d} \beta_{n}(s)\right|^{2}\right) \leq C(\tau) e^{-2 \alpha \mathrm{N}}\|b\|_{\mathrm{G}_{\alpha}}^{2} .
$$

Putting it all together, we obtain by Gronwall's inequality:

$$
\begin{aligned}
\mathbf{E}\left(\sup _{t \in(0, \tau]}\left\|u(t)-u^{\mathrm{N}}(t)\right\|_{\mathcal{H}}^{2}\right) & \leq C_{m}\left(\int_{0}^{\tau} \mathbf{E}\left(\sup _{s \in(0, t]}\left\|u(s)-u^{\mathrm{N}}(s)\right\|_{\mathcal{H}}^{2}\right) \mathrm{d} t+\tau^{2} \mathrm{~h}^{2}+\mathrm{N}^{-m}\right) \\
& \leq C_{m}(\tau)\left(\mathrm{h}^{2}+\mathrm{N}^{-m}\right) .
\end{aligned}
$$

Jensen's Inequality then gives Proposition 3.3.

\section{Higher Order Schemes}

The easiest way to obtain higher order schemes is to apply an Itô-Taylor expansion (see [12]). For example:

$$
u_{n}(\mathrm{~h})=e^{-\mathrm{h} n^{2}} u_{n}(0)+\int_{0}^{\mathrm{h}} e^{-(\mathrm{h}-s) n^{2}} F_{n}(u(s)) \mathrm{d} s+\int_{0}^{\mathrm{h}} e^{-(\mathrm{h}-s) n^{2}} b_{n} \mathrm{~d} \beta_{n}(s)
$$




$$
\begin{gathered}
=e^{-\mathrm{h} n^{2}} u_{n}(0)+e^{-\mathrm{h} n^{2}} \mathrm{~h} F_{n}(u(0)) \\
+\int_{0}^{\mathrm{h}}\left(\int _ { 0 } ^ { s } e ^ { - ( \mathrm { h } - \sigma ) n ^ { 2 } } \left(n^{2} F_{n}(u(\sigma))+\sum_{j} \partial_{j} F_{n}(u(\sigma))\left(-j^{2} u_{j}(\sigma)+F_{j}(u(\sigma))\right)\right.\right. \\
\left.\quad+\frac{1}{2} \sum_{j} b_{j}^{2} \partial_{j}^{2} F_{n}(u(\sigma))\right) \mathrm{d} \sigma \\
\left.+\int_{0}^{s} e^{-(\mathrm{h}-\sigma) n^{2}} \sum_{j} b_{j} \partial_{j} F_{n}(u(\sigma)) \mathrm{d} \beta_{j}(\sigma)\right) \mathrm{d} s \\
+\int_{0}^{\mathrm{h}}\left(e^{-\mathrm{h} n^{2}}+\int_{0}^{s} n^{2} e^{-(\mathrm{h}-\sigma) n^{2}} \mathrm{~d} \sigma\right) b_{n} \mathrm{~d} \beta_{n}(s) .
\end{gathered}
$$

Using the same discretisation procedure as in Section 3 (projecting onto a finite set of modes and replacing $\sigma$ by 0 ) we get

$$
\begin{aligned}
& u_{n}((k+1) \mathrm{h}) \\
& =e^{-\mathrm{h} n^{2}}\left(u_{n}(k \mathrm{~h})+\mathrm{h}\left(1+\frac{1}{2} \mathrm{~h} n^{2}\right) F_{n}(u(k \mathrm{~h}))\right. \\
& \quad+\frac{1}{2} \mathrm{~h}^{2} \sum_{j=-\mathrm{N}}^{\mathrm{N}}\left(\partial_{j} F_{n}(u(k \mathrm{~h}))\left(-j^{2} u_{j}(k \mathrm{~h})+F_{j}(u(k \mathrm{~h}))\right)+\frac{1}{2} b_{j}^{2} \partial_{j}^{2} F_{n}(u(k \mathrm{~h}))\right) \\
& +\frac{1}{\sqrt{3}} \mathrm{~h}^{3 / 2} \sum_{j=-\mathrm{N}}^{\mathrm{N}} b_{j} \partial_{j} F_{n}(u(k \mathrm{~h})) Z_{k+1, j} \\
& \left.+b_{n} \sqrt{\mathrm{h}}\left(1+\mathrm{h} n^{2}\right) X_{k+1, n}-b_{n} n^{2} \frac{1}{\sqrt{3}} \mathrm{~h}^{3 / 2} Z_{k+1, n}\right),
\end{aligned}
$$

where

$$
Z_{k, n}=\sqrt{3} \mathrm{~h}^{-3 / 2}\left(\int_{(k-1) \mathrm{h}}^{k \mathrm{~h}} \beta_{n}(s) \mathrm{d} s\right)
$$

are $\mathcal{N}(0,1)$ random variables. They are not independent of the variables $X_{k, n}$ :

$$
\mathbf{E}\left(X_{k, n} Z_{k, n}\right)=\frac{1}{2} \mathrm{~h}^{2}
$$

We refer to [12], p.19, for a method to generate these correlated Gaussian variables. The scheme obtained in Eq.(6.1) has strong order 3/2. Other schemes, both weak and strong, may be derived in the standard way [12].

\section{Numerical Results}

We have implemented the scheme Eq. (3.2) and, for comparison, the implicit Euler-Maruyama method of Eq. (3.5) for the Ginzburg-Landau nonlinearity $F_{\mathrm{GL}}(u)=-|u|^{2} u$. In practice, for the nonlinear term we compute the coefficients $F_{n}\left(u^{\mathrm{N}}(k \mathrm{~h})\right)$ by first applying the inverse 
(a)

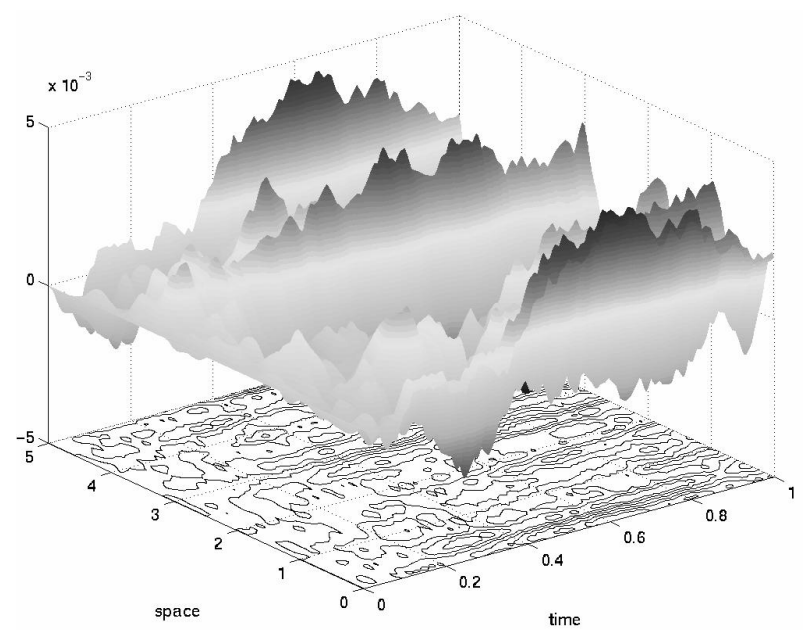

(b)
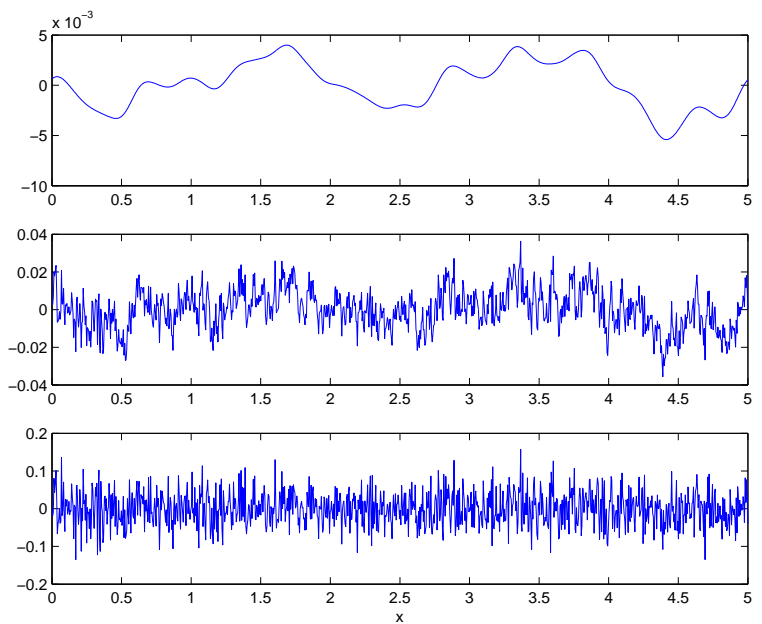

Figure 1: (a) A sample path of Gevrey noise with $b_{n}=\exp (-n), n \neq 0$. In (b) we plot sample noise at $t=1$ (top) Gevrey noise, (middle) noise with $b_{n}=n^{-1}, n \neq 0$, (bottom) noise with no decay in the coefficients $b_{n}=1$ corresponding to spatially white noise.

fast Fourier transform to $u^{\mathrm{N}}(k \mathrm{~h})$, computing $F(u)$ in real space followed by the fast Fourier transform.

We examine the regularity of solutions and strong convergence for smooth (Gevrey) noise and non-smooth (non-Gevrey) noise. In Figure 1 (a) we have plotted a sample noise path which satisfies (2.6), for which we have taken $b_{n}=\exp (-\mu|n|), n \neq 0$. The spatial smoothness of the noise is parameterized by $\mu$, and in this example $\mu=1$. In (b) we compare at $t=1$ the Gevrey noise (top) against noise with $b_{n}=n^{-1}, n \neq 0$ (middle) and against noise with constant values of $b_{n}=1$, spatially white noise (bottom). Neither $b_{n}=n^{-1}$ or $b_{n}=1$ satisfies the hypothesis (2.6). In each case we took the same seed for the random number generator, $2^{10}$ Fourier coefficients and a time step of $h=0.01$. Figure 1 shows the smooth spatial nature of the forcing satisfying (2.6) (although it is white noise in time).

In Figures 2-4 we plot sample solutions (top) and the corresponding (semilog) of the Fourier spectrum (bottom). The computations for these figures used $2^{11}$ Fourier modes. In Figure 2 smooth noise was taken with two different time steps, in (a) $\mathrm{h}=10^{-4}$ and in (b) $\mathrm{h}=10^{-3}$. In (a) we see that the spectrum decays exponentially with wave number where as in (b) for large wave numbers we see a faster decay in the spectrum. This is can be understood from the factor $\exp \left(-\mathrm{h} n^{2}\right)$ in Eq. (3.2), for exponential decay in the spectrum we require $\mathrm{h}$ to scale like $N^{-1}$. As we have seen in Eq.(3.7), at each time $u(t)$ is the solution of the linear heat equation, which is known to be an entire analytic function. Thus, our numerical scheme consists in approximating the Gevrey analytic solution $u(t)$ of the PDE (which possibly has a singularity in the complex plane) by a sequence of entire analytic functions $u^{\mathrm{h}<r N}(t)$, which have increasingly rapid growth along the imaginary axis as we let $\mathrm{h}$ go to 0 (but no singularity).

In contrast to Figure 2 the solutions of Figure 3(a) and (b) were computed with nonsmooth noise (a) $b_{n}=n^{-1}$ and (b) $b_{n} \equiv 1$. For smooth noise the spectrum decays exponen- 
tially in wave number (in accordance with the theory) and comparing Figures 2 and 3 we see much slower decay in the spectrum for the non-smooth noise.

Figure 4 shows similar calculations for the implicit Euler-Maruyama scheme with smooth (a) and non-smooth noise, $b_{n}=n^{-1}, n \neq 0$ in (b). Although we have not proved that implicit Euler-Maruyama has solutions in a Gevrey class of regularity with smooth forcing, these results indicate that this is probably the case. The non-smooth case is similar to that observed in Figure 3.

We also tested strong convergence numerically (see Figures 5-6). To approximate a "true" solution we computed a solution with $2^{12}$ Fourier modes and $\mathrm{h}=10^{-5}$. In Figure 5 (a) the average of the $\mathrm{L}_{\text {per }}^{2}([0,2 \pi), \mathbb{C})$-norm of the error is displayed for 10 orbits against $h$. Each curve corresponds to a different number of Fourier modes $\mathrm{N}=2^{q}$ with $q=7,8,9,10,11,12$ and a reference straight line of slope 1 is shown for comparison. In Figure 5 (b) we have taken smooth noise. We observe that the order of convergence is consistent with the theory. Figure 5 (b) shows similar calculations for the case of $b_{n}=n^{-1}$, so (2.6) is no longer satisfied (and the noise is no longer in a Gevrey space of regularity). Comparing Figure 5 (a) and (b) we see that the scheme exhibits stiffness for non-smooth noise and is less accurate.

In Figure 6 (a) we show the results of the same calculations performed for the implicit Euler-Maruyama scheme of Eq. (3.5) with smooth noise. We see qualitatively the same behaviour as the scheme given by (3.2) with smooth noise, shown in Figure 5 (a). In Figure 6 (b) we show the results of the standard Euler-Maruyama scheme of Eq. (3.4) with smooth noise, and again we observe qualitatively the same behaviour as the scheme given by (3.2) of Figure 5 (a). However, the standard Euler-Maruyama scheme Eq. (3.4) has worse stability properties (see $[7,8]$ ), and a restriction is required that $\mathrm{h}<2 / \mathrm{N}^{2}$. As a consequence, this scheme is unlikely to be used for practical computations. This stability constraint meant that the "true" solution was found with $\mathrm{h}=10^{-6}$ and $2^{10}$ modes and we show computations performed with $2^{7}$ modes and $\mathrm{h}=2 \times 10^{-6}, 5 \times 10^{-6}, 10^{-5}, 2.5 \times 10^{-5}, 5 \times 10^{-6}, 10^{-4}$. Like the implicit Euler-Maruyama scheme we have no result on the regularity of the solution for the standard Euler-Maruyama with smooth noise.

Similar numerical results are found for the case of multiplicative noise

$$
\dot{u}(t)=\Delta u(t)+F(u(t))+u(t) \mathcal{Q W}(t) .
$$

We adapt the scheme (3.2) and consider

$$
u_{n}^{\mathrm{N}}((k+1) \mathrm{h})=e^{-\mathrm{h} n^{2}}\left(u_{n}^{\mathrm{N}}(k \mathrm{~h})+\mathrm{h} F_{n}\left(u^{\mathrm{N}}(k \mathrm{~h})\right)+d_{n} \sqrt{\mathrm{h}} X_{k+1, n}\right),
$$

where $d_{n}$ are the Fourier coefficients of $u \mathcal{Q} \dot{W}$. In figure 7 we have plotted in (a) the solution and spectrum using this scheme for $\mathrm{N}=2^{11}$ and $h=10^{-4}$. In (b) we have plotted the solution and Fourier modes for the multiplicative noise case using the implicit Euler-Maruyama method. In Figure 8 (a) we examine convergence of the scheme (7.2) with multiplicative noise with $2^{11}$ Fourier modes. In (b) we have looked at convergence for the the implicit Euler-Maruyama scheme with multiplicaitve noise. The average of the $\mathrm{L}_{\text {per }}^{2}([0,2 \pi), \mathbb{C})-$ norm of the error is displayed for 5 orbits against $h$. The reference solution was computed with $\mathrm{h}=10^{-6}$ and $2^{12}$ Fourier modes. A reference line with slope 1 is also shown. Numerically we observe the same convergence rate as in the additive noise case and that the Euler-Maruyama and the scheme (7.2) have similar properties. 


\section{Acknowledements}

We would like to thank James Robinson and Tony Shardlow for comments on an earlier draft. The work of Jacques Rougemont was supported by the EPSRC GR/R29949/01.

\section{References}

[1] J. Bricmont, A. Kupiainen, And R. Lefevere, Probabilistic estimates for the two-dimensional stochastic Navier-Stokes equations, J. Statist. Phys., 100 (2000), pp. 743-756.

[2] I. CHuESHOv, Gevrey regularity of random attractors for stochastic reaction-diffusion equations, Random Operators and Stoch. Equations, 8 (2000), pp. 143-162.

[3] G. DA Prato And J. Zabczyk, Stochastic equations in infinite dimensions, Cambridge University Press, Cambridge, 1992.

[4] A. Doelman And E. Titi, Regularity of solutions and the convergence of the Galerkin method in the complex Ginzburg-Landau equation, Numer. Func. OPt. Anal., 14 (1993), pp. 299-321.

[5] A. B. FerRari AND E. S. Titi, Gevrey regularity for nonlinear analytic parabolic equations, Commun. Part. Diff. Eqs., 23 (1998), pp. 1-16.

[6] C. Foias And R. TEMAM, Gevrey class regularity for the solutions of the NavierStokes equation, J. Differential Equations, 87 (1989), pp. 359-369.

[7] W. GRecksch And P. Kloeden, Time-discretized Galerkin approximations of parabolic stochastic pdes, Bull. Australian Math. Soc., 54 (1996), pp. 79-85.

[8] E. Hausenblas, Approximation for semilinear stochastic evolution equations, Potential Analysis, (2002).

[9] D. J. Higham, X. MAO, AND A. STUART, Strong convergence of euler-type methods for nonlinear stochastic differential equations, SIAM J. Num. Anal., 40 (2002), pp. 1041-1063.

[10] O. Kallenberg, Foundations of modern probability, Springer-Verlag, New York, 1997.

[11] P. KLOEDEN AND S. SHOTT, Linear-implicit strong schemes for Ito-Galerkin approximations of stochastic pdes, J. Appl. Math. Stoch. Anal, (2001).

[12] P. E. Kloeden And E. Platen, Numerical solution of stochastic differential equations, Springer-Verlag, Berlin, 1992.

[13] G. J. LORD AND J. Rougemont, Topological and $\varepsilon$-entropy for large volume limits of discretised parabolic equations, SIAM J Num. Anal., 40 (2002), pp. 1311-1329.

[14] G. J. LORD AND A. M. STUART, Discrete Gevrey regularity, attractors and uppersemicontinuity for a finite difference approximation to the complex Ginzburg-Landau equation, Num. Func. Anal. Opt., 16 (1995), pp. 1003-1049.

[15] X. MAO, Stochastic Differential Equations and Applications, Horwood, 1997. 
[16] J. C. Mattingly, The Stochastic Navier-Stokes Equation: Energy Estimates and Phase Space Contraction, PhD thesis, Princeton University, 1998.

[17] J. C. Mattingly, The dissipative scale of the stochastic Navier Stokes equation: Regularization and analyticity, J. Statistical Physics, 108 (2002), pp. 1157-1179.

[18] K. S. Promislow, Time analyticity and Gevrey class regularity for solutions of a class of dissipative partial differential equations, Nonlinear Anal.:Theor. Meth. Appl., 16 (1991), pp. 959-980.

[19] A. SHIRIKYAN, Analyticity of solutions for randomly forced two-dimensional NavierStokes equations, Russian Math. Surveys., 57 (2002), pp. 785-799.

[20] P. TAKÁČ AND A. JÜNGEL, A nonstiff Euler discretization of the complex GinzburgLandau equation in one space dimension, SIAM J. Numer. Anal., 38 (2000), pp. 292328. 
(a)
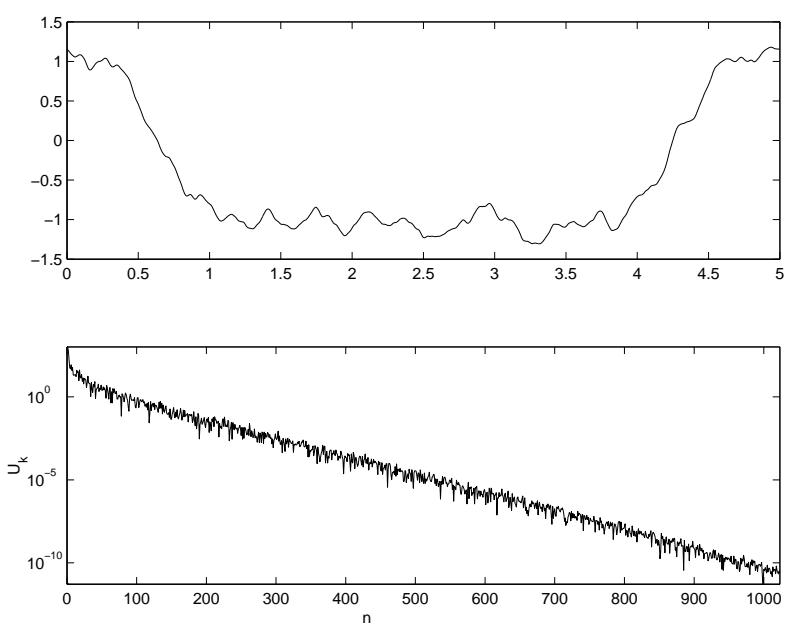

(b)
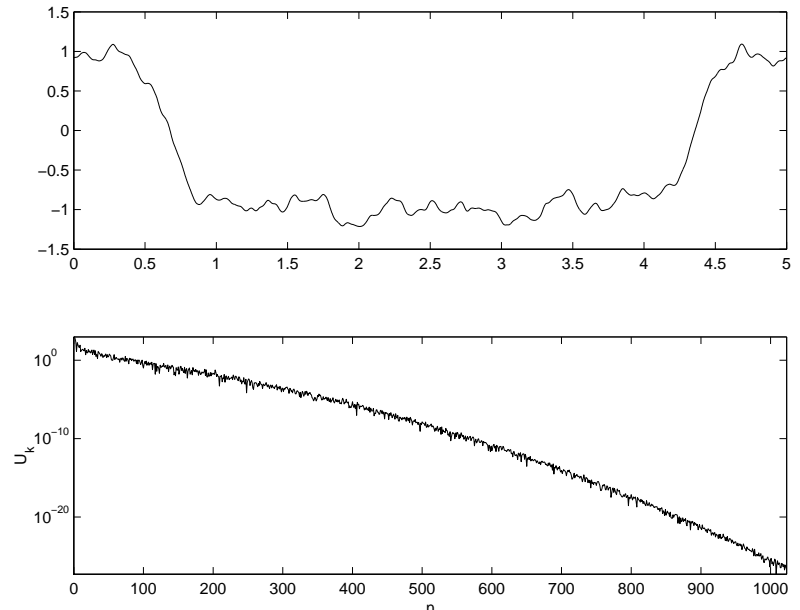

Figure 2: Solution at $t=1$ (top) and spectrum (below) using scheme (3.2) with smooth noise. Computed with $2^{11}$ modes and step size (a) $\mathrm{h}=10^{-4}$ and (b) $\mathrm{h}=10^{-3}$.

(a)
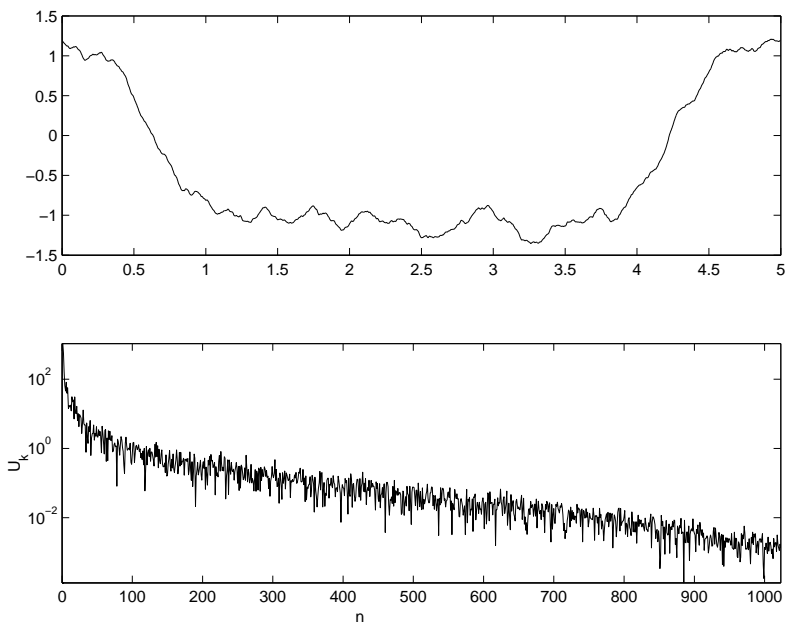

(b)
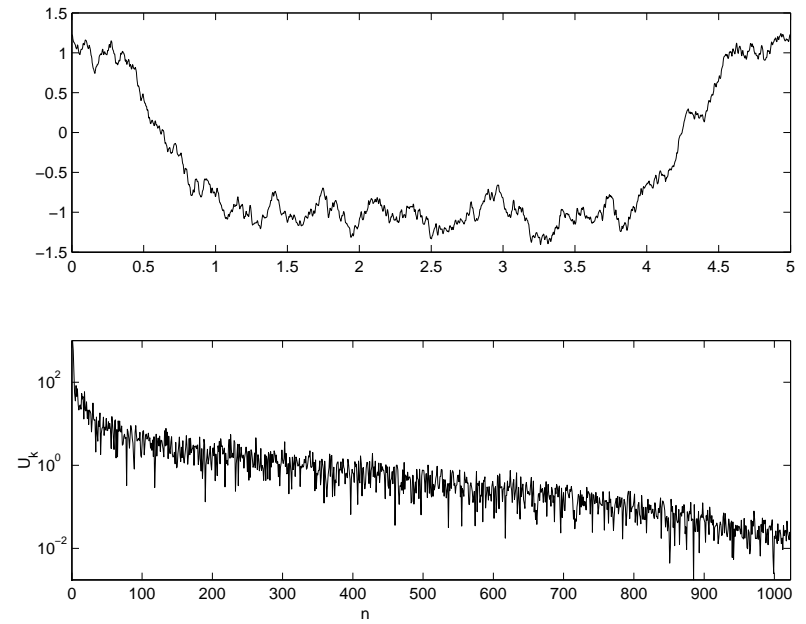

Figure 3: Solution at $t=1$ (top) and spectrum (below) using the scheme (3.2) with non-smooth noise (a) $b_{n}=n^{-1}$ and (b) $b_{n} \equiv 1$. Computed with $2^{11}$ modes and with $\mathrm{h}=10^{-4}$. 
(a)
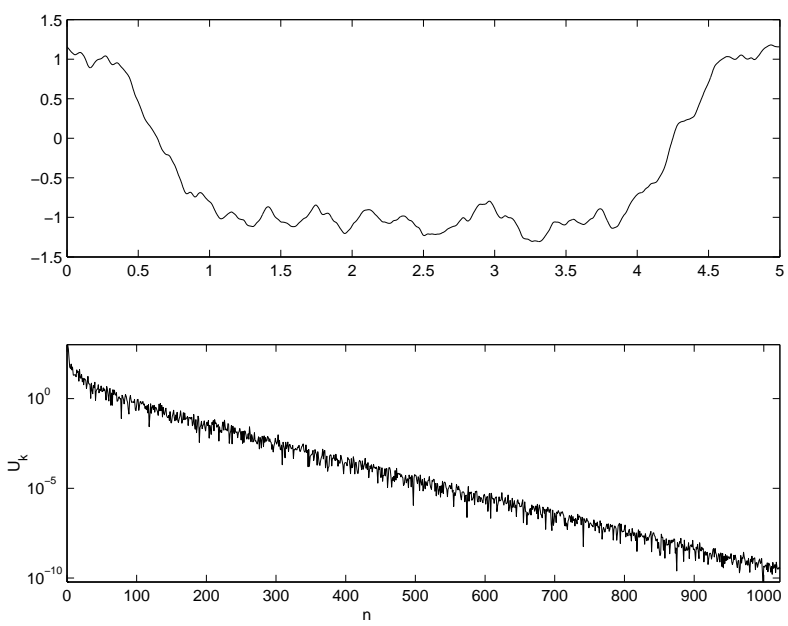

(b)
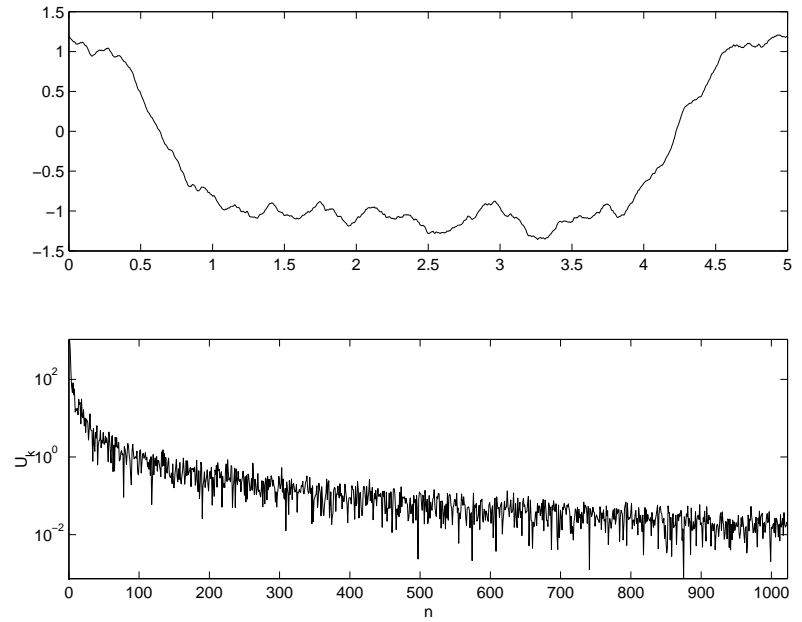

Figure 4: Solution at $t=1$ (top) and spectrum (below) using the implicit Euler-Maruyama scheme (3.5) with (a) smooth noise and (b) non-smooth noise $\left(b_{n}=n^{-1}\right)$. Solution computed with $2^{11}$ modes $\mathrm{h}=10^{-4}$.

(a)

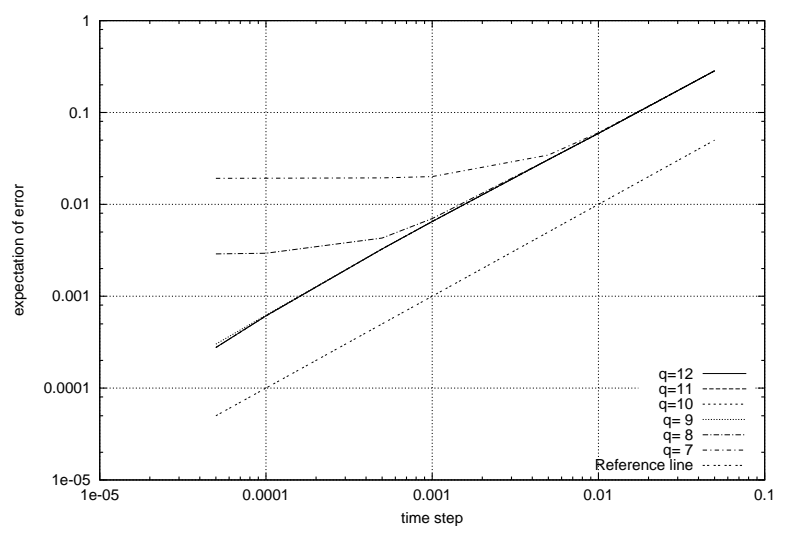

(b)

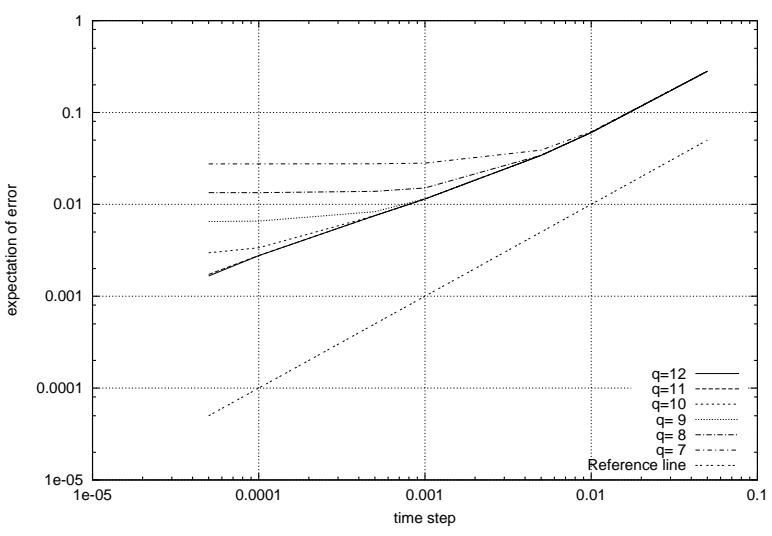

Figure 5: Strong error estimates for (a) smooth noise and (b) non-smooth noise for the scheme (3.2) with different values of $\mathrm{h}$ and $\mathrm{N}=2^{q}, q=7,8,9,10,11,12$. Also shown in each case is a reference line of slope 1 . 
(a)

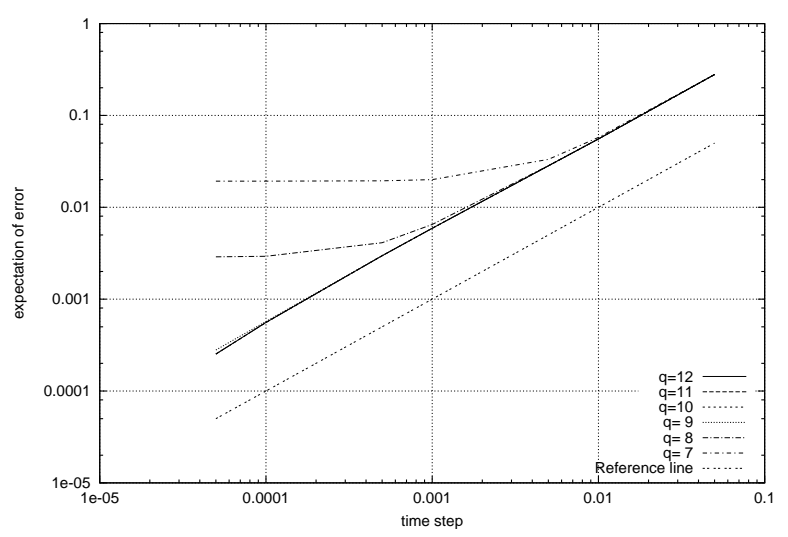

(b)

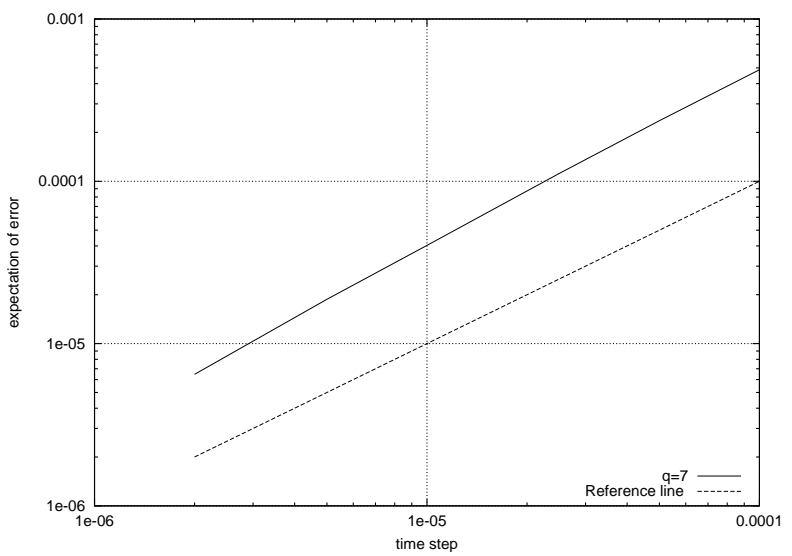

Figure 6: Strong error estimates with smooth noise for (a) the implicit Euler-Maruyama scheme (3.5) $\left(\mathrm{N}=2^{q}, q=7,8,9,10,11,12\right)$ and (b) the standard Euler-Maruyama scheme (3.4) $(\mathrm{N}=$ $2^{7}$ ). For stability reasons the reference solution for the (b) was computed with $\mathrm{h}=10^{-6}$ and $\mathrm{N}=2^{10}$. Also shown in each case is a reference line of slope 1 .

(a)
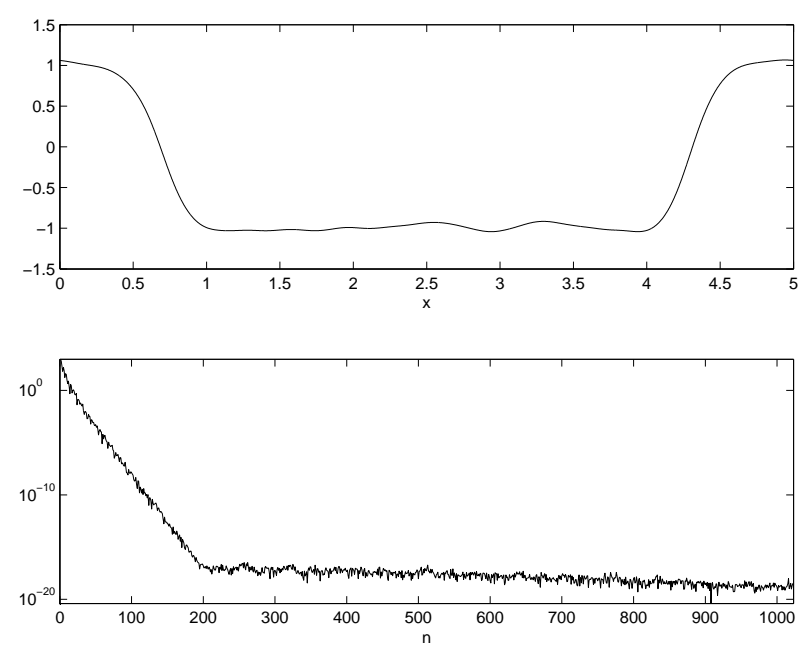

(b)
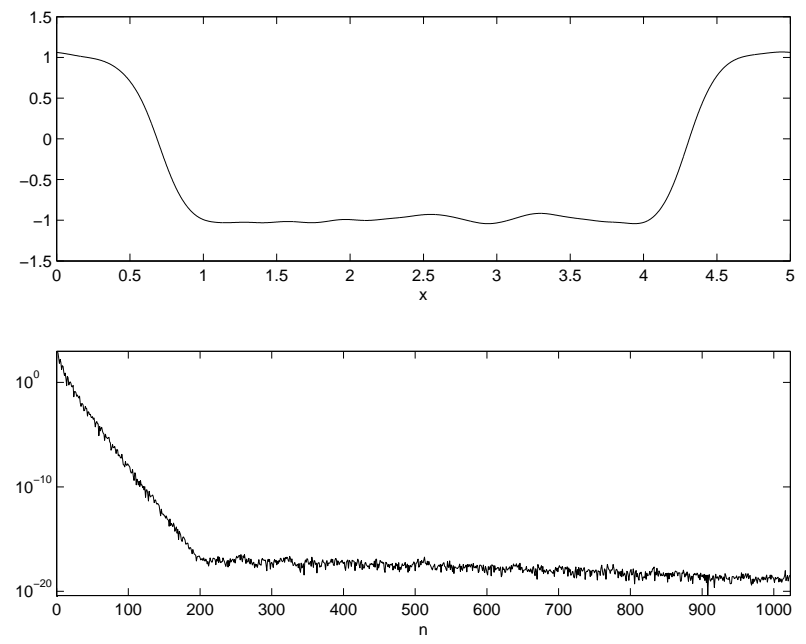

Figure 7: Scheme (7.2) (a) and implicit Euler-Maruyama (b) with smooth multiplicative noise. Solutions shown at $t=1$ computed with $2^{11}$ modes and $h=10^{-4}$ (top) in space and (bottom) in Fourier space. 
(a)

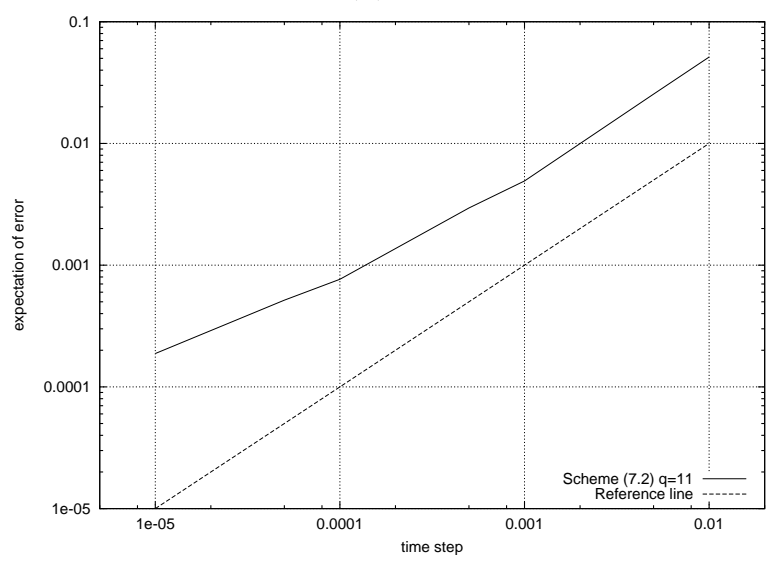

(b)

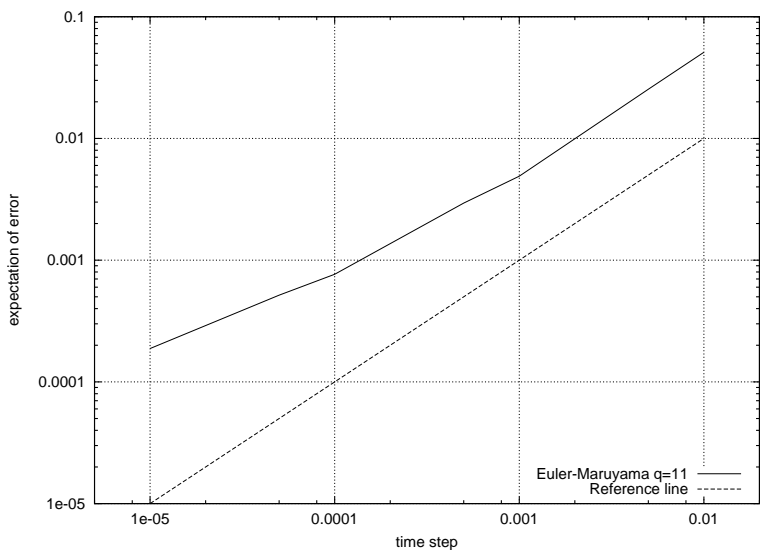

Figure 8: Illustration of convergence (a) of the scheme (7.2) for smooth multiplicative noise and (b) the implicit Euler-Maruyama scheme. In each case the reference line has slope 1. 